\title{
Identification and Evaluation of a New Tumor Cell-Binding Peptide, FROP-1
}

\author{
Sabine Zitzmann ${ }^{1,2}$, Susanne Krämer ${ }^{2}$, Walter Mier ${ }^{2}$, Ulrike Hebling ${ }^{1,2}$, Annette Altmann ${ }^{1,2}$, Axel Rother ${ }^{4}$, \\ Dietmar Berndorff ${ }^{5}$, Michael Eisenhut ${ }^{3}$, and Uwe Haberkorn ${ }^{1,2}$ \\ ${ }^{I}$ Clinical Cooperation Unit Nuclear Medicine, German Cancer Research Center, Heidelberg, Germany; ${ }^{2}$ Department of Nuclear \\ Medicine, University of Heidelberg, Heidelberg, Germany; ${ }^{3}$ Department of Radiopharmaceutical Chemistry, German Cancer \\ Research Center, Heidelberg, Germany; ${ }^{4}$ Forschungszentrum Dresden-Rossendorf, Dresden, Germany; and ${ }^{5}$ Bayer-Schering-Pharma \\ Research Laboratories, Berlin, Germany
}

Peptides are useful tools for the targeted delivery of radionuclides or chemotherapeutic drugs to their site of action within an organism. Given that the peptide receptor is overexpressed at the tumor, therapeutically active doses can be delivered to the tumor with reduced side effects. Because currently known peptides are restricted to a small number of tumors, new molecules and their corresponding receptors have to be identified to enlarge the spectrum of malignancies that can be diagnosed or treated using tumor-targeting peptides. Methods: A 12-amino-acid peptide phage display system was applied to identify a new peptide binding to follicular thyroid carcinoma cells. The properties of the radiolabeled peptide were assessed in binding, competition, and internalization experiments in a variety of tumor cell lines including FRO82-2 and MCF-7 cells, and the pharmacokinetic behavior of the radiolabeled peptide was evaluated in tumor-bearing mice. Peptide stability was studied in human serum. Results: After 5 selection rounds, the new peptide, FROP-1 (EDYELMDLLAYL), was identified. It showed binding to follicular thyroid carcinoma as well as anaplastic thyroid carcinoma, mammary carcinoma, cervix carcinoma, prostate carcinoma, and cell lines derived from head and neck tumors, and low affinity could be observed to control cells such as human umbilical vein endothelial cells or immortalized keratinocytes. In MCF7 cells, $78 \%$ and $86 \%$ of the bound activity was internalized after 10 and 60 min of incubation, respectively. Stability experiments in human serum showed the appearance of a degradation product after $15 \mathrm{~min}$. Tumor uptake of the radioactive labeled peptide increased for $45 \mathrm{~min}$ in nude mouse models, reaching an accumulation level of approximately 3.6 percentage injected dose (\%ID)/g for FRO82-2 tumors or approximately $3.8 \% \mathrm{ID} / \mathrm{g}$ for MCF-7 tumors. Conclusion: The target of FROP-1 is most likely a molecule found generally in tumors, making this peptide highly attractive for diagnostic or therapeutic applications. However, modifications are needed to increase stability and affinity.

Key Words: tumor targeting; thyroid carcinoma; mammary carcinoma; peptide; phage display

J Nucl Med 2007; 48:965-972

DOI: 10.2967/jnumed.106.036699

\footnotetext{
Received Sep. 26, 2006; revision accepted Mar. 6, 2007.

For correspondence or reprints contact: Sabine Zitzmann, PhD, Bayer Schering Pharma Research Laboratories, Müllerstrasse 178, 13342, Berlin, Germany.

E-mail: sabine.zitzmann@schering.de

COPYRIGHT @ 2007 by the Society of Nuclear Medicine, Inc.
}

$\mathbf{T}$ reatment of thyroid cancer is successful if the tumor accumulates radioiodine or is surgically resectable. Because radiation therapy and chemotherapy are usually not effective, tumors that lose their ability to concentrate iodine represent a clinical problem (1). Redifferentiation trials, such as through administration of retinoic acid, sometimes show iodide uptake (2). The reason seems to be related to the expression of nuclear receptors for retinoic acid in thyroid carcinoma-receptors that are shown in varying amounts in different cell lines. However, it is unclear whether the amount of isotope accumulation is sufficient for a therapeutically useful radiation dose. Up to $70 \%$ of thyroid cancers express somatostatin receptors, which can be used for treatment with ${ }^{90} \mathrm{Y}$-DOTA-Tyr ${ }^{3}$-octreotide (3). At present, the clinical value of this therapy in these patients has not been fully evaluated. Furthermore, tumors showing weak or no somatostatin receptor expression are not amenable to this type of treatment.

The choice of target is an important prerequisite to specific targeting of a tumor with a radiolabeled antibody or peptide (4). Only if the receptor or target molecule is overexpressed on the tumor can appropriate amounts of the targeting molecule be accumulated. Several peptide receptors are known to be specific for certain types of tumors (5). Autoradiographic studies have shown that the cholecystokinin-B/ gastrin receptors are overexpressed in more than $90 \%$ of metastatic medullary thyroid cancer, in a high percentage of cases of small cell lung cancer, in stromal ovarian cancer, and potentially in a variety of other tumors, including gastrointestinal adenocarcinomas, neuroendocrine tumors, and malignant glioma $(6,7)$. Therefore, several cholecystokinin/ gastrin-related peptides have been investigated for their targeting properties to improve staging of metastatic medullary thyroid cancer, which is sometimes difficult to stage with conventional radiologic procedures (8). Another example is the gastrin-releasing peptide (bombesin) receptor, which is overexpressed in a variety of human cancers (e.g., breast, prostate, pancreatic, and small cell lung) (9). The list of known peptide receptors also covers the vasoactive 
intestinal peptide receptors (gastrointestinal stromal tumor and other stromal tumors), neurotensin receptors, and neuropeptide $\mathrm{Y}$ receptors (breast cancer and ovarian tumors) (10). However, for many tumors these targets are not applicable.

Phage display technology is a promising tool for the identification of new binders with high specificity. The method is used for several applications, such as mapping and mimicking of epitopes, identifying new receptors and natural ligands, identifying high-affinity antibodies and analogs, isolating specific antigens that bind to bioactive compounds, producing novel enzyme inhibitors and DNAbinding proteins, and probing cellular and tissue-specific processes (11). Phage display was successfully applied to identify novel peptides with high specificity targeting a variety of molecules or structures such as tumor neovasculature (12) or to identify specifically binding peptides for human lung tumor cells (13). In this study, we investigated the use of a phage peptide library to select a peptide specific for thyroid carcinoma, which was then evaluated for stability, for binding to various cell lines, and for its biodistribution in tumor-bearing mice.

\section{MATERIALS AND METHODS}

\section{Cell Lines}

All cell lines were cultivated at $37^{\circ} \mathrm{C}$ in a $5 \% \mathrm{CO}_{2}$ incubator. The human thyroid follicular carcinoma cell line FRO82-2 (University of California, Los Angeles) was cultivated in RPMI 1640 with GlutaMAX containing $10 \%$ fetal calf serum, $0.15 \%$ sodium bicarbonate, $1.5 \mathrm{mM}$ sodium pyruvate, $1.5 \times$ nonessential amino acids (all: Invitrogen), and $25 \mathrm{mM} \mathrm{N}$-(2-hydroxyethyl)piperazine$N^{\prime}$-(2-ethanesulfonic acid) (HEPES). The human breast cancer cell line MDA-MB 435 (National Cancer Institute), the human prostate cancer cell lines PC-3 and DU-145 (both: American Type Culture Collection), the human embryonic kidney cell line 293 (American Type Culture Collection), the human anaplastic thyroid carcinoma cell line SW1736 (obtained from Nis-Erik Heldin, Uppsala, Sweden), and the human colorectal carcinoma cell line HCT-116 (American Type Culture Collection) were cultured in RPMI 1640 with GlutaMAX, containing 10\% fetal calf serum and $25 \mathrm{mM}$ HEPES. The human breast cancer cell line MCF 7 (Tumorbank, German Cancer Research Center); the cervix carcinoma cell line HelaTeton transfected with the Tet-expression system (Becton Dickinson); the thyroid cell line FTC-133 (European Collection of Cell Cultures); the human squamous cell carcinoma cell lines HNO 97, HNO 210, HNO 223, and HNO 237 (Dr. Charles Herold-Mende, University Hospital Heidelberg, Germany (14)); and the human colon adenocarcinoma cell line SW948 (German Collection of Microorganisms and Cell Cultures [DSMZ], Braunschweig, Germany) were cultured in DMEM with GlutaMAX, containing $10 \%$ fetal calf serum and $25 \mathrm{mM}$ HEPES. The human colon adenocarcinoma cell line Caco-2 (DSMZ) was cultured in DMEM/F-12 medium with GlutaMAX, containing $10 \%$ fetal calf serum and $25 \mathrm{mM}$ HEPES. The human HPV16 immortalized keratinocyte cell line HPV16-GM (Dr. Pascal Tomakidi, University Hospital Heidelberg, Germany) was cultured in keratinocyte growth medium 2 with supplements (PromoCell). Human umbilical vein endothelial cells (HUVECs) were isolated as described in the literature (15). HUVEC cultivation was performed on gelatin (1\%)-coated cell culture flasks using medium 199 (Invitrogen) containing 20\% fetal calf serum, $2 \mathrm{mM}$ glutamine, $100 \mathrm{IU} / \mathrm{mL}$ penicillin, $100 \mathrm{IU} / \mathrm{mL}$ streptomycin, and 2 ng bFGF/mL (Roche Diagnostics).

\section{Selection of Tumor Cell-Binding Peptides}

The phage display library used was a linear 12-amino-acid peptide library (Ph.D.-12; New England Biolabs). Each selection round was conducted as follows: $10^{11}$ transducing units were added to cell line 293 cells for a negative selection. After $1 \mathrm{~h}$, the medium was collected and centrifuged for $5 \mathrm{~min}$ at $1,500 \mathrm{rpm}$, and the supernatant was transferred to FRO82-2 cells grown to $90 \%$ confluency. After $1 \mathrm{~h}$, the cells were washed 4 times with $10 \mathrm{~mL}$ of Hank's balanced salt solution(+) (Invitrogen) plus 1\% bovine serum albumin (BSA) and 4 times with $10 \mathrm{~mL}$ of Hank's balanced salt solution(-) plus $1 \%$ BSA. The cells were then detached with $4 \mathrm{~mL}$ of phosphate-buffered saline (PBS) plus $1 \mathrm{mM}$ ethylenediaminetetraacetic acid for $5 \mathrm{~min}$ and centrifuged for $5 \mathrm{~min}$ at $1,500 \mathrm{rpm}$. The cell pellet was washed 3 times in $1 \mathrm{~mL}$ of Hank's balanced salt solution(-) plus 1\% BSA and lysed with $1 \%$ Triton X-100. Ten microliters of the lysate were used to titer the phages. The remaining lysate was amplified in $50 \mathrm{~mL}$ of ER2537 bacteria according to the manufacturer's protocol. For the next selection round, $10^{11}$ transducing units from the previous selection round were used. Five selection rounds were performed, followed by single-stranded DNA isolation from clones (QIAprep Spin M13 Kit; Qiagen Inc.). Sequencing of the DNA allowed the identification of the corresponding peptide.

\section{Peptide}

The FROP-1 peptide (EDYELMDLLAYL) was obtained by solid-phase peptide synthesis using Fmoc chemistry. Radiolabeling was achieved by iodination using the chloramine-T method (16). The labeled peptide was purified by high-performance liquid chromotography (HPLC) on a $5-\mu \mathrm{m}, 250 \times 4 \mathrm{~mm}$ column (LiChrosorb RP-select B; Merck) using Tris/phosphate and methanol as eluents. The specific activities obtained were 90 $\mathrm{GBq} / \mu \mathrm{mol}$ for the ${ }^{125} \mathrm{I}$-labeled peptide and $110 \mathrm{GBq} / \mu \mathrm{mol}$ for the ${ }^{131}$ I-labeled peptide.

\section{In Vitro Binding Experiments}

For binding studies, 400,000 cells (FRO82-2) or 200,000 cells (MCF-7) were seeded into 6-well plates and cultivated for $24 \mathrm{~h}$. The medium was replaced by $1 \mathrm{~mL}$ of fresh medium (without fetal calf serum). ${ }^{125}$ I-labeled peptide was added to the cell culture (1-2 $\times$ $10^{6} \mathrm{cpm} / \mathrm{well}$ ) and incubated for the appropriate times, varying from $1 \mathrm{~min}$ to $4 \mathrm{~h}$. When the competitor was used, unlabeled peptide $\left(10^{-4} \mathrm{M}-10^{-11} \mathrm{M}\right)$ was preincubated for $30 \mathrm{~min}$. After incubation, the cells were washed 3 times with $1 \mathrm{~mL}$ of PBS and subsequently lysed with $0.5 \mathrm{~mL}$ of $0.3 \mathrm{M} \mathrm{NaOH}$. Radioactivity was determined with a $\gamma$-counter and calculated as percentage applied dose per $10^{6}$ cells. If BSA was used as a blocking agent, it was added to a final concentration of $1 \%$ in medium without fetal calf serum.

\section{Cell Counting}

Immediately after the completion of an in vitro binding experiment, the number of cells in 3 reference wells was determined. The wells were washed with PBS before $1 \mathrm{~mL}$ of ethylenediaminetetraacetic acid-PBS solution $(1 \mathrm{mM})$ was added to detach the cells. Cell aggregations were disrupted by pipetting, and the 1-mL cell suspension was added to $20 \mathrm{~mL}$ of cell-counting liquid 
(Coulter Isoton II diluent; Beckman-Coulter). Five hundred microliters of the suspension were counted 3 times in a cell counter (Coulter-Counter; Beckman-Coulter), multiplied by 42 to obtain the cell number per well, and the mean value of the 3 reference wells was calculated.

\section{Stability Experiments}

One hundred microliters of ${ }^{125}$ I-labeled peptide (approximately $10^{7} \mathrm{cpm}$ ) were added to a tube containing $1 \mathrm{~mL}$ of human serum and incubated at $37^{\circ} \mathrm{C}$. At various time points, $100 \mu \mathrm{L}$ were removed and the proteins precipitated with $100 \mu \mathrm{L}$ of acetonitrile. The solution was centrifuged for $5 \mathrm{~min}$ at $13,000 \mathrm{rpm}$, and the cleared lysate was analyzed by reversed-phase HPLC with a $0 \%-100 \%$ gradient $\mathrm{MeCN}$ in $30 \mathrm{~min}$. For the analysis of peptide fragment mass, $200 \mu \mathrm{g}$ of unlabeled FROP-1 peptide were incubated for $1 \mathrm{~h}$ at $37^{\circ} \mathrm{C}$ in human serum, serum proteins were precipitated, and peaks were isolated and analyzed by matrix-assisted laser desorption/ ionization time-of-flight (MALDI-TOF) mass spectrometry (17). The molecular integrity of the ${ }^{125}$ I-labeled peptide in the cell supernatant was determined by HPLC analysis of medium samples from the incubation with FRO82-2 or MCF-7 cells at various time points as described above.

\section{Animals and Tumor Growth}

Female 6-wk-old BALB/c $n u / n u$ mice were obtained from Charles River WIGA and housed in VentiRack ventilated cage racks (BioZone). For inoculation of the tumors in nude mice, a Matrigel matrix (BD Biosciences) cell suspension $\left(5 \times 10^{6}\right.$ cells) was injected subcutaneously into the anterior region of the mouse trunk. Tumors were grown to a size of approximately $1.0 \mathrm{~cm}^{3}$. All animals were cared for according to the national animal guidelines.

\section{Organ Distribution with Radioiodinated FROP-1}

${ }^{131}$ I-FROP-1 was intravenously injected in the tail vein of female $n u / n u$ mice $\left(2.8 \times 10^{7} \mathrm{cpm} /\right.$ mouse $)$ carrying the subcutaneously transplanted human tumors FRO82-2 or MCF7. At 5, 15, 45, and 135 min after injection, the mice were sacrificed. The organs were removed and weighed, and the radioactivity was determined using an automated $\mathrm{NaI}(\mathrm{Tl})$ well counter (CobraII; Canberra Packard). Thereafter, the percentage injected dose per gram of tissue (\%ID/g) was calculated. For the in vivo competition experiment, $500 \mu \mathrm{g}$ of unlabeled FROP-1 were coinjected with the ${ }^{131}$ I-FROP-1 and the mice were sacrificed after $15 \mathrm{~min}$.

\section{RESULTS}

\section{Isolation of Peptide Binding to Cell Line FR082-2 by Peptide Phage Display}

For identification of tumor-specific peptides, phages expressing 12 mer peptides on their surface were applied to in vitro selection rounds on FRO82-2 cells. Each individual selection round consisted of approximately $10^{11}$ transducing units of the phages, which were added to a cell culture dish with cell line 293 cells for a negative selection for $1 \mathrm{~h}$, followed by positive selection on FRO82-2 cells also for $1 \mathrm{~h}$. The unbound phages were washed off, and bound phages were recovered by lysing the FRO82- 2 cells. After 5 selection rounds, single-phage clones were isolated and amplified, and single-stranded DNA was obtained for sequencing. Among 20 clones sequenced, 95\% of all peptides showed the same sequence: EDYELMDLLAYL (FROP-1).

\section{Cell Line Binding Profile}

Binding studies were performed on a variety of tumor cells to evaluate the binding potential of FROP-1. For the binding experiments, FROP-1 was prepared by Fmoc solidphase synthesis, labeled with ${ }^{125} \mathrm{I}$, and used for the subsequent binding experiments. The highest binding capacity for ${ }^{125}$ I-FROP-1 - with up to $6 \%$ of the applied dose per $10^{6}$ cells - was found for the breast cancer cell line MCF-7, which was inhibited by more than $95 \%$ by an excess of unlabeled FROP-1 (Fig. 1). An array of various tumor cell lines also showed increased binding of ${ }^{125}$ I-FROP-1, such as HeLaTeton with $3.9 \%$ cell uptake, PC-3 with $2.7 \%$, HNO237 with $3.2 \%$, SW1736 with $2.2 \%$, CaCo-2 with $1.4 \%$, MDA-MB435 and DU-145 with $1.3 \%$, HNO210 with $1.2 \%$, HNO97 and HCT116 with $1.1 \%$, and HNO223 with $1.0 \%$. The primary endothelial cells HUVEC and the benign keratinocyte cell line HPV16-GM showed only $0.8 \%$ cell uptake, which was inhibited to less than $65 \%-$ $70 \%$ by an excess of unlabeled FRO-1. No binding was observed in FTC133 and SW948 cells.

\section{Binding Kinetics and Competition of FROP-1 to a Thyroid and a Mammary Tumor Cell Line}

Because FRO82-2 and MCF-7 cells showed the highest binding capacity, the peptide was further characterized in these cell lines. FRO 82-2 and MCF-7 cells were incubated for $1 \mathrm{~h}$ with the ${ }^{125}$ I-labeled peptide (Fig. 2A) and, for competition experiments, with unlabeled peptide as the competitor $\left(10^{-4} \mathrm{M}\right)$. Radioactivity of the lysed cells was calculated as percentage applied dose per $10^{6}$ cells. For the FRO82-2 thyroid tumor cells, a cell uptake of up to $2.8 \%$ - and for the MCF-7 cells, an uptake of up to $6 \%$ - could be observed after $1 \mathrm{~h}$ and could be inhibited by the addition of an excess of unlabeled FROP-1 but not by an excess of unlabeled octreotide peptide at the same concentration (Fig. 2B). In vitro internalization studies were performed to determine the rate of internalization of ${ }^{125}$ I-FROP-1 in MCF-7 cells (Fig. 2C). To distinguish between surface-bound peptide and internalized peptide, 2 acidic wash steps were included in the washing procedure to remove all outside-bound peptide. After a 10-min incubation of the cells with ${ }^{125} \mathrm{I}$-FROP- 1 at $37^{\circ} \mathrm{C}$, the internalized radioactivity was measured as $5 \%$ cell uptake (representing $78 \%$ of total cell-bound activity) and increased to almost $6 \%$ cell uptake (representing $86 \%$ of total cell-bound activity) after $1 \mathrm{~h}$ of incubation. The presence of unlabeled peptide prevented uptake into the cells.

For evaluation of the time course of peptide binding, ${ }^{125}$ I-labeled peptide was added to FRO82-2 and MCF-7 cells and incubated for times ranging from 5 min to $4 \mathrm{~h}$. After incubation, the cells were lysed and the radioactivity as percentage applied dose per $10^{6}$ cells was calculated (Fig. 3A). Binding of FROP-1 to MCF-7 cells was rapid, with the highest binding rate obtained after $5 \mathrm{~min}$, followed by a decrease until $1 \mathrm{~h}$ after the start of the experiment, 

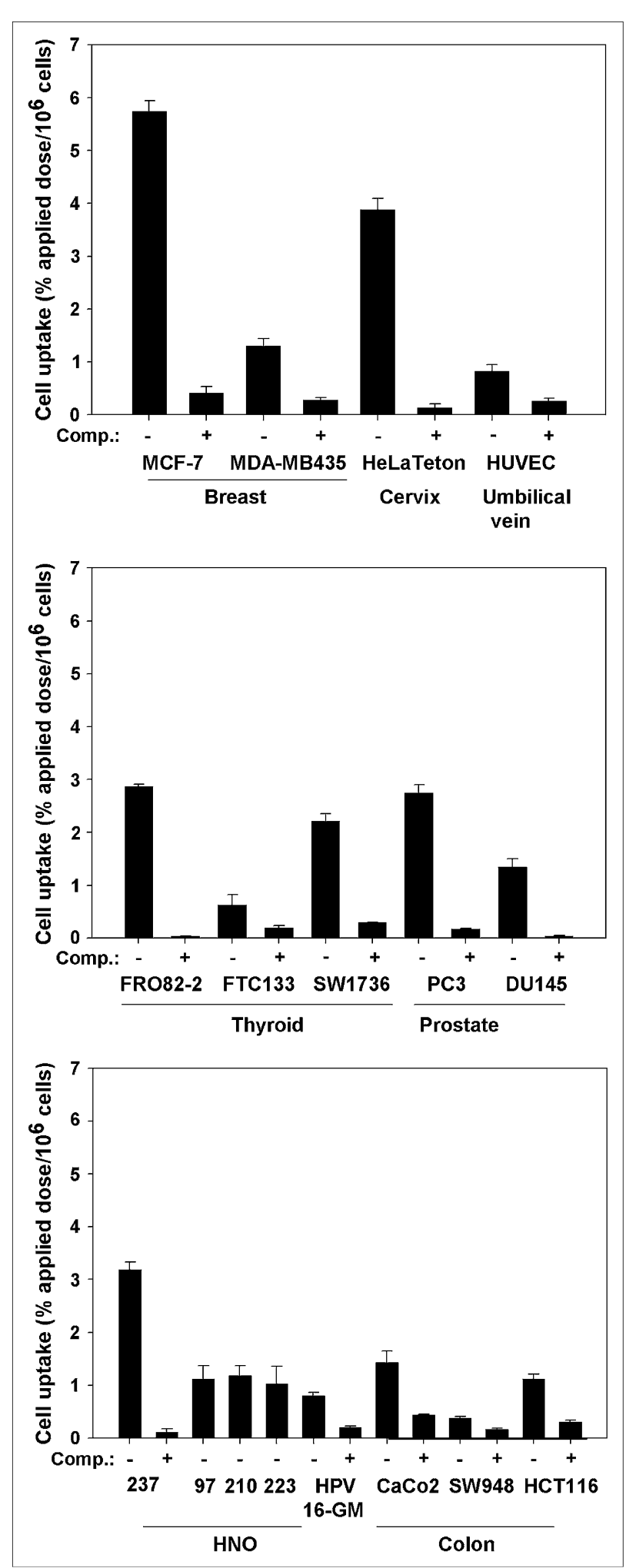

FIGURE 1. In vitro binding studies to various tumor cell lines-MCF-7, MDA-MB435, HeLaTeton, FR082-2, FTC133, SW1736, PC-3, DU-145, HNO237, HNO97, HNO210, HNO223, CaCo-2, SW948, and HCT116 - and to nontumor cell line HPV16-GM and primary endothelial cells HUVEC. Cells were incubated either with ${ }^{125} \mathrm{I}-\mathrm{FROP}-1$ without competitor $(-)$ or in presence of $10^{-4} \mathrm{M}$ unlabeled FROP-1 (+) for $1 \mathrm{~h}$ at $37^{\circ} \mathrm{C}$. Experiments were performed in triplicate; SDs are shown. when a steady-state level of about $6 \%$ applied dose per $10^{6}$ cells was reached. The binding kinetics of FROP-1 to FRO82-2 cells were different. Accumulation was slower, reaching a maximum after $30 \mathrm{~min}$, followed by a slow decrease of bound radioactivity. The presence of $1 \%$ BSA in the incubation medium did not lead to a reduction but rather to an increase in binding capacity of up to $3 \%$ cell uptake for FRO82-2 cells, which have slower binding kinetics, and to a markedly slower binding for MCF-7 cells (Fig. 3B). Competition experiments using unlabeled FROP-1 as the competitor in concentrations between $10^{-4}$ and $10^{-7} \mathrm{M}$ showed 2 different $\mathrm{IC}_{50}$ for FRO82-2 and MCF-7 cells: The value for FRO82-2 cells was approximately $8 \mu \mathrm{M}$, and that for MCF-7 cells was about $12 \mu \mathrm{M}$ (Fig. 3C). The peptide stability in the medium for the in vitro assays was assessed by HPLC analysis. The incubation with FRO82-2 cells led to a half-life of the peptide in the supernatant of approximately $30 \mathrm{~min}$, and the incubation with MCF-7 cells led to a half-life of 15 min (Fig. 4). In both cell types, the degradation resulted in the formation of ${ }^{125}$ I-labeled tyrosine as proven by analysis of a ${ }^{125} \mathrm{I}-$ tyrosine sample. The incubation of the peptide in cell-free medium did not show this degradation, and the major event observed was deiodination.

\section{Serum Stability of FROP-1}

The stability of the ${ }^{125}$ I-FROP-1 peptide was tested to predict potential cleavage sites within the peptide sequence. During incubation of the labeled peptide in human serum at $37^{\circ} \mathrm{C}$, aliquots were removed, serum proteins precipitated with acetonitrile, and the samples analyzed by HPLC (Fig. 5). The purified peptide had a retention time of $17.6 \mathrm{~min}$. After 5 min, a small second peak was visible with a retention time of $17.92 \mathrm{~min}$, which increased until it reached the approximate size of peak 1 after $15 \mathrm{~min}$. After $30 \mathrm{~min}$ of incubation time, peak 1 had almost disappeared and 2 more degradation products appeared at 4.05 and $9.13 \mathrm{~min}$. After $120 \mathrm{~min}$ of incubation time, the full-length peptide was almost completely degraded, whereas the products at 4.05 and $9.13 \mathrm{~min}$ were still visible. The serum stability experiment was also performed with a larger amount of unlabeled peptide to allow the isolation of peaks and subsequent analysis by MALDITOF. Two peaks that were isolated after $1 \mathrm{~h}$ of incubation time, eluting closely together, probably corresponded to the peaks at 17.6 and $17.92 \mathrm{~min}$ of the radiolabeled peptide stability experiment. Indeed, the mass of the earlier peak was 1,509.84 (1,486.74 [full-length peptide] plus 23 [1 sodium atom]), and the peak appearing later had a mass of 1,380.23, which is most likely the full-length peptide minus the $\mathrm{N}$-terminal glutamate plus 23 for 1 sodium atom. A much smaller peak eluting earlier with a mass of 550.35 could not be attributed to a distinct peptide fragment.

\section{Biodistribution of Radiolabeled FROP-1}

The distribution of FROP-1 was investigated in vivo: The peptide was labeled with ${ }^{131} \mathrm{I}$ and intravenously injected 


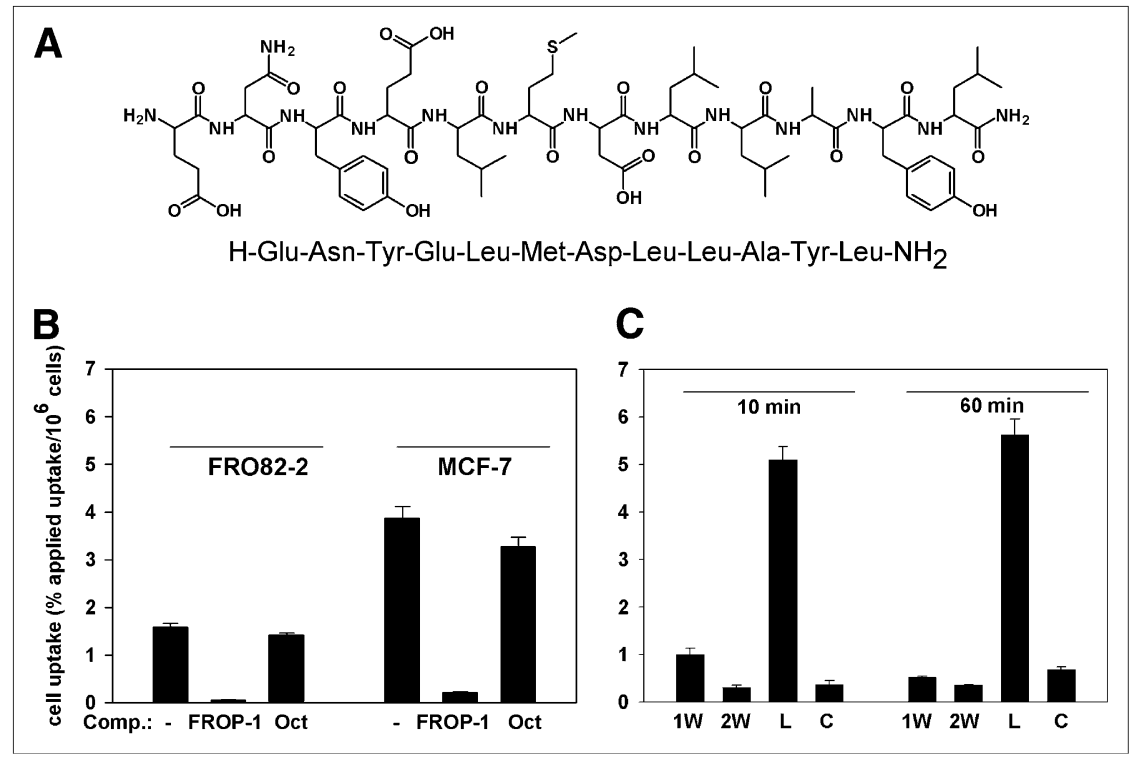

FIGURE 2. (A) Amino acid sequence of peptide FROP-1 isolated by peptide phage display. (B) In vitro binding assay with FROP-1. FRO82-2 and MCF-7 cells were grown for $24 \mathrm{~h}$. ${ }^{125} \mathrm{I}-\mathrm{FROP}-1$ was added to wells and incubated for $1 \mathrm{~h}$; as competitor, either $1 \times 10^{-4} \mathrm{M}$ unlabeled FROP-1 or octreotide (Oct) was added immediately before incubation with labeled FROP-1. (C) Internalization of 125I-FROP-1 in MCF-7 cells. Cells were incubated with 1-2 $\times 10^{6} \mathrm{cpm}$ radioligand for $10 \mathrm{~min}$ and $1 \mathrm{~h}$ at $37^{\circ} \mathrm{C}$. After being washed with acidic glycine buffer $(\mathrm{pH}$ 2.8), cells were lysed and internalized radioactivity was measured. Experiments were performed in triplicate; SDs are shown. $1 \mathrm{~W}=$ first acidic wash, $2 \mathrm{~W}=$ second acidic wash, $\mathrm{L}=$ lysate, $\mathrm{C}=$ control with unlabeled peptide; Comp. = competitor.

into female $n u / n u$ mice carrying human thyroid tumors (FRO82-2) or human mammary carcinoma (MCF-7). Biodistribution in mice carrying the FRO82-2 tumor showed that FROP-1 accumulated in the tumor to a level of 1.6 $\% \mathrm{ID} / \mathrm{g}$ after $5 \mathrm{~min}$, increased to $3.1 \% \mathrm{ID} / \mathrm{g}$ after $15 \mathrm{~min}$ and to $3.8 \% \mathrm{ID} / \mathrm{g}$ after $45 \mathrm{~min}$, and decreased to $2.7 \% \mathrm{ID} / \mathrm{g}$ at $135 \mathrm{~min}$ (Fig. 6A). This biodistribution is in contrast to all other organs, which showed a decrease of radioactivity over the entire experiment. The kidneys showed the highest amount of radioactivity, $15 \% \mathrm{ID} / \mathrm{g}$ at $5 \mathrm{~min}$, but a rapid decrease to $6.7 \% \mathrm{ID} / \mathrm{g}$ after $15 \mathrm{~min}$ and to $2.7 \% \mathrm{ID} / \mathrm{g}$ after $45 \mathrm{~min}$. Radioactivity cleared from the blood more slowly, with a decrease from $6.7 \% \mathrm{ID} / \mathrm{g}$ after $5 \mathrm{~min}$ to $5.8 \% \mathrm{ID} / \mathrm{g}$ after $15 \mathrm{~min}$ and to $5.4 \% \mathrm{ID} / \mathrm{g}$ after $45 \mathrm{~min}$. After $135 \mathrm{~min}$, most of the peptide had left the organs. In experiments with MCF-7 tumors, the level of radioactivity in the tumor also increased, from $1.9 \% \mathrm{ID} / \mathrm{g}$ after $5 \mathrm{~min}$ to $2.4 \% \mathrm{ID} / \mathrm{g}$ after 15 $\mathrm{min}, 3.6 \% \mathrm{ID} / \mathrm{g}$ after $45 \mathrm{~min}$, whereas radioactivity in the liver sharply decreased from $8.0 \% \mathrm{ID} / \mathrm{g}$ at $5 \mathrm{~min}$ to 1.3 $\% \mathrm{ID} / \mathrm{g}$ at $45 \mathrm{~min}$ (Fig. 6B). Also, all other organs showed a decrease in radioactivity with time. For both tumor models, the thyroid showed a strong accumulation of radioactivity of up to $95 \% \mathrm{ID} / \mathrm{g}$ after $45 \mathrm{~min}$. The tumor-to-muscle ratios for both the FRO82-2 tumors and the MCF-7 tumors showed an increase over the entire experiment (Fig. 6C). The tumor-to-muscle ratio for FRO82-2 tumors increased from 0.8 after 5 min to 4.8 after $135 \mathrm{~min}$; MCF-7 tumors showed an increase from 2.2 after 5 min to 7.9 after 135 min. To exclude a possible contribution of Matrigel to tracer uptake, we assessed the accumulation of FROP-1 in FRO82-2 and MCF-7 tumors inoculated with or without Matrigel and observed no significant difference between tumors inoculated with Matrigel and tumors inoculated in PBS (Fig. 6D). For an in vivo competition experiment, $500 \mu \mathrm{g}$ of unlabeled FROP-1 were coinjected with ${ }^{131}$ I-FROP-1 into mice bearing FRO82-2 tumors (Table 1). In most
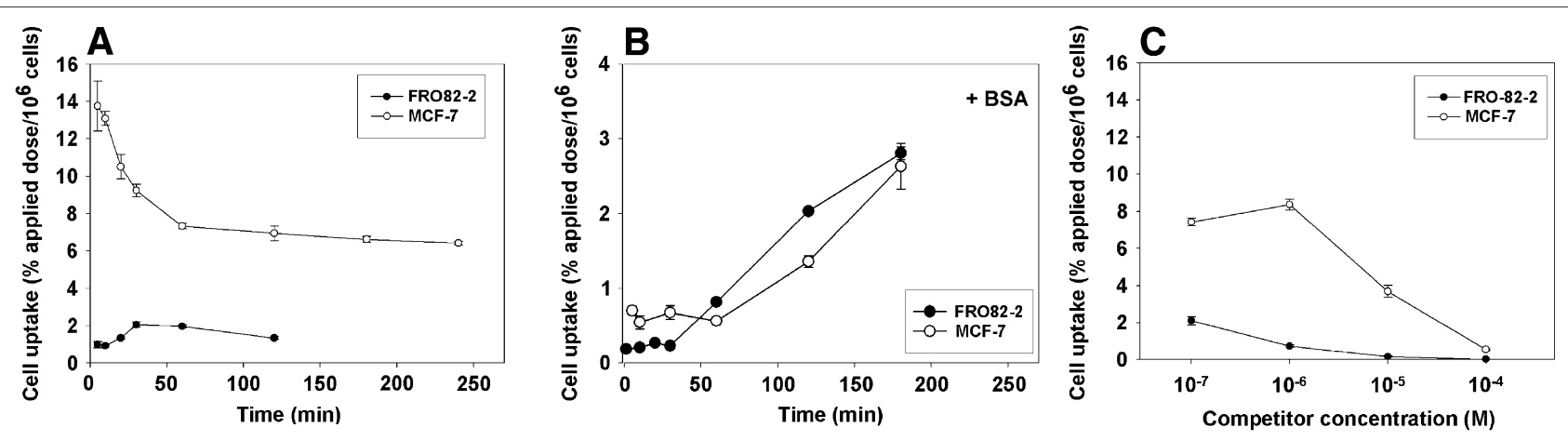

FIGURE 3. (A and B) In vitro binding kinetics of FROP-1. FRO82-2 and MCF-7 cells were grown for $24 \mathrm{~h} .{ }^{125}$ I-FROP-1 was added and incubated for $5,10,15,30,60,120,180$, and 240 min without (A) or with (B) 1\% BSA. (C) In vitro competition assay with FROP-1 using various competitor concentrations. FRO82-2 and MCF-7 cells were grown for 24 h. Unlabeled FROP-1 in concentrations ranging from $10^{-4} \mathrm{M}$ to $10^{-7} \mathrm{M}$ were added to cells immediately before ${ }^{125} \mathrm{~F}-\mathrm{FROP}-1$ and incubated for $1 \mathrm{~h}$. Experiments were performed in triplicate; SDs are shown. 
FIGURE 4. HPLC analysis of $125 /-$ FROP-1 in supernatant of FRO82-2 (A) and MCF-7 (B) cells at different time points. Final product of degradation is 125I-tyrosine in both cases (peak eluting at approximately $1.55 \mathrm{~min}$ ).

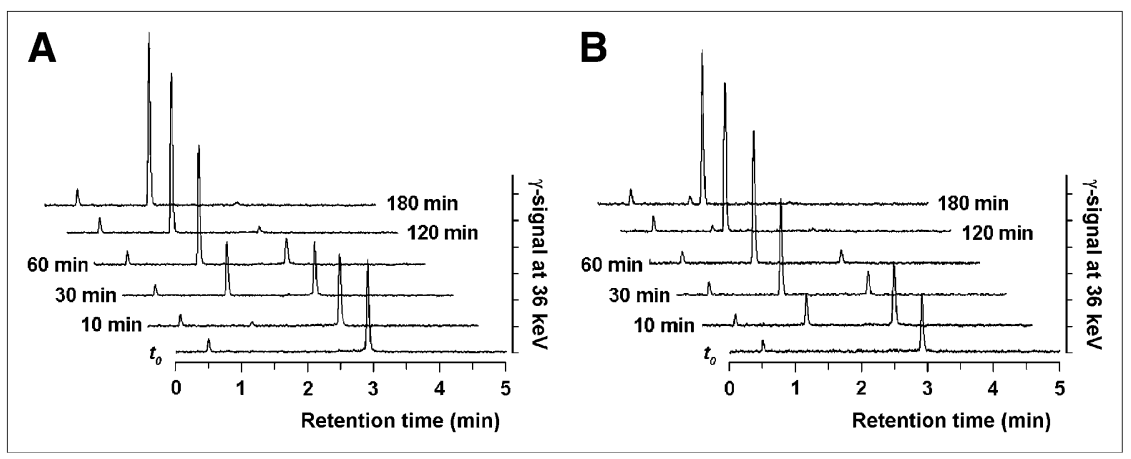

\section{DISCUSSION}

Using the peptide phage display system on the thyroid cancer cell line FRO82-2, we identified a new tumor-binding peptide, FROP-1. Use of the Basic Linear Alignment and Search Tool against protein databases (e.g., SwissProt) did not show any sequence identity of FROP-1 to known peptide ligands. In particular, no similarity to cholecystokinin-B/ gastrin was found, suggesting that FROP-1 is not a derivative of cholecystokinin-B/gastrin or its family. No inference can be drawn as to the receptor to which the new peptide binds. Because we used a black box approach by incubating whole tumor cells instead of defined proteins, further work is needed to identify the target molecule. On the other hand, this black box approach offers the opportunity to find new structures useful for diagnosis and treatment (18).

The peptide bound to the thyroid cancer cells FRO82-2, and this interaction can be inhibited by more than $95 \%$ by the unlabeled peptide itself but not by the presence of the unspecific competitor octreotide. The breast cancer cells MCF-7 bound the peptide FROP-1 even better, with up to 7\% applied dose per $10^{6}$ cells, and this binding could also be inhibited by the unlabeled FROP-1 and was not inhibited by octreotide. An internalization experiment in which all cell surface-bound radioactive peptide was removed by acidic washes revealed a rapid uptake into the cells that increased slightly over time. This finding may be due to the fact that the panning procedure with a relatively long exposure $(60 \mathrm{~min})$ of the phages to the cells rather favored the selection of motifs leading to internalization. The fact that there was no further increase of internalized FROP-1 is perhaps best explained by the results of the stability experiments, which showed a degradation of the peptide by the cells over time that was more prominent for the MCF-7 cells than for the FRO82-2 cells and might explain the differences observed in the binding kinetics (19).

The kinetics of FROP-1 binding between the 2 cell lines were markedly different. MCF-7 initially showed high radioactivity bound to the cells, with a steep decrease for the first hour and then a steady state over time. FRO82-2 showed a much slower accumulation, with a lower peak at 30 min followed by a slow decrease over time. This initial accumulation phase was slowed by the addition of BSA to the incubation medium, resulting in an initial lag-phase of 


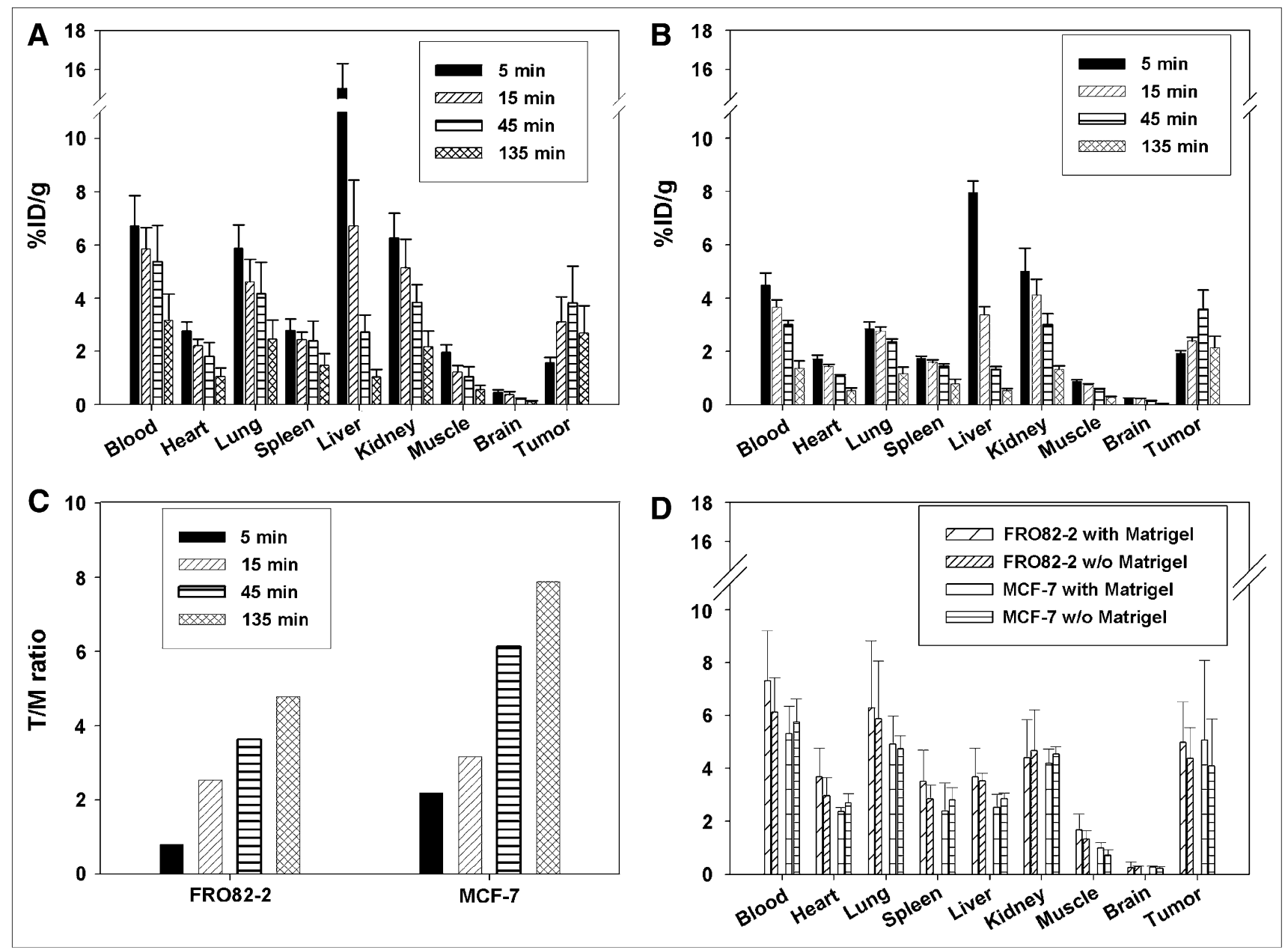

FIGURE 6. Biodistribution of FROP-1 in female BALB/c nu/nu mice carrying FRO82-2 tumors ( $n=3$ animals per time point) (A) or MCF-7 tumors ( $n=6$ animals per time point) (B). Animals received intravenous injection of ${ }^{131} \mid \mathrm{I}-\mathrm{FROP}-1$, and radioactivity was measured in tumor and control organs after 5, 15, 45, and $135 \mathrm{~min}$. SEM is shown. (C) Tumor-to-muscle ratios calculated for FRO82-2 and MCF-7 tumors at various time points. (D) Biodistribution of ${ }^{125}$ I-FROP-1 at 45 min comparing FRO82-2 and MCF-7 tumors incubated with and without Matrigel (BD Biosciences) ( $n=3$ animals per time point).

30 min before the FRO82-2 and MCF-7 cells showed any bound activity. But the level of activity associated with the FRO82-2 cells reached the same height irrespective of the presence or absence of BSA. A possible explanation for this initial lag-phase could be an unspecific binding of FROP-1 to BSA resulting in an albumin-bound peptide pool from which the peptide was slowly released and could bind to the cells. Because the BSA concentration was $1 \%$, there was a large excess of BSA molecules over ${ }^{125}$ I-FROP-1 molecules, which could easily result in complete quenching of all free radiolabeled peptides. This could also explain the high accumulation of the FROP-1 peptide in the liver during the in vivo biodistribution experiments.

FROP-1 binds not only to the follicular thyroid carcinoma cells used for biopanning but also to a variety of other tumor entities including anaplastic thyroid carcinoma, mammary carcinoma, cervix carcinoma, prostate carcinoma, and cell lines derived from head and neck tumors, although to a lesser extent. This variety indicates that the target of FROP-1 is a molecule found in tumors generally rather than only in thyroid carcinoma, making this molecule highly attractive for diagnostic or therapeutic applications.

The radiolabeling of FROP-1 at its 2 tyrosines will most likely result in the formation of several products. The HPLC diagrams, especially after the radiolabeling with ${ }^{131}$ I, showed up to 4 peaks in variable proportions, whereas the radiolabeling with ${ }^{125}$ I resulted mostly in 1 major peak that could be purified by HPLC. The FROP-1 peptide contains, in addition to 2 tyrosines, methionine, which can become oxidized under radiolabeling conditions. Therefore, the 4 peaks are probably an iodinated primary tyrosine with either an oxidized or a reduced methionine and an iodinated secondary tyrosine with either an oxidized or a reduced methionine. It is unlikely that both tyrosines will be iodinated in the same peptide during the labeling procedure. This mixture of different products is difficult to separate and was used whole for the in vivo experiments. Because the iodine label, as well as the oxidized methionine, is within the molecule, 
TABLE 1

In Vivo Competition of FROP-1

\begin{tabular}{lc}
\hline Site & Ratio \\
\hline Blood & 1.04 \\
Heart & 1.02 \\
Lung & 1.07 \\
Spleen & 1.13 \\
Liver & 1.26 \\
Kidney & 1.02 \\
Muscle & 0.87 \\
Brain & 0.87 \\
Tumor & 1.31
\end{tabular}

Ratio $=$ ratio of organ without competitor to organ with competitor.

Data are from 3 female BALB/c nu/nu mice carrying FRO82-2 tumors and injected intravenously either with ${ }^{131}$ I-FROP-1 alone or with ${ }^{131} \mathrm{I}-\mathrm{FROP}-1$ plus $500 \mu \mathrm{g}$ of unlabeled FROP-1. Radioactivity was measured in tumor and control organs after $15 \mathrm{~min}$.

the possibility is high that one or the other will affect binding of the peptide to the receptor and might even change secondary structure. An appropriate experimental setting to examine this influence would be a mutational analysis with exchanges of the 3 affected amino acids. Another possible solution would be the use of a chelator to avoid labeling or oxidation of amino acids within the targeting peptide.

The stability experiment indicated that degradation of the peptide begins with the release of the first $\mathrm{N}$-terminal amino acid (glutamate). MALDI-TOF analysis of the other HPLC peaks did not lead to a convincing mass analogy as to allow an estimate of the cleaved amino acids. Possible is not only a simple cleavage of peptide binds within the peptides but also a modification through serum enzymes. Exchange of this amino acid by a $\mathrm{D}$ amino acid or methylation may improve the stability of the molecule, because it is possible that the glutamate might not be needed for binding or that stabilization of this first cleavage site might prevent further cleavage or result in different cleavage products. Also, methionine and tyrosine can be used for optimization and to identify the important amino acids and structural requirements for binding. Furthermore, synthesis of FROP-1 multimers may improve not only stability and labeling efficiency but also affinity and accumulation in tumors (20).

\section{CONCLUSION}

Because of its binding to a variety of tumor cells, internalization, and relatively stable accumulation in tumors in vivo, FROP-1 is a promising molecule for the diagnosis and therapy of tumors such as thyroid and mammary carcinoma and possibly also for head and neck or cervix carcinoma. However, the radiolabeling, stability, and affinity of the molecule need improvement, which can be done through exchange of amino acids, coupling of chelators such as tetraazacyclododecanetetraacetic acid, or multimerization.

\section{ACKNOWLEDGMENTS}

We acknowledge the contribution of Sigrid Peschke to the in vitro experiments and Helmut Eskerski, Ursula Schierbaum, and Karin Leotta to the animal experiments. We especially thank Kerstin Schmidt for sharing her HUVEC cells, Christa Herold-Mende for providing the HNO cell lines, and Pascal Tomakidi to providing the HPV16-GM cell line. Additionally, we thank Andreas Kolbe for information technology support and Markus Wolf for his suggestion concerning the use of Matrigel. This work was supported by grants HA2901/5-1 and 5-2 from the DFG.

\section{REFERENCES}

1. Fagin JA. How thyroid tumors start and why it matters: kinase mutants as targets for solid cancer pharmacotherapy. J Endocrinol. 2004;183:249-256.

2. Gruning T, Tiepolt C, Zophel K, Bredow J, Kropp J, Franke WG. Retinoic acid for redifferentiation of thyroid cancer: does it hold its promise? Eur $J$ Endocrinol. 2003;148:395-402.

3. Bodei L, Handkiewicz-Junak D, Grana C, et al. Receptor radionuclide therapy with ${ }^{90}$ Y-DOTATOC in patients with medullary thyroid carcinomas. Cancer Biother Radiopharm. 2004;19:65-71.

4. Reubi JC, Macke HR, Krenning EP. Candidates for peptide receptor radiotherapy today and in the future. $J$ Nucl Med. 2005;46(suppl):67S-75S.

5. de Jong M, Kwekkeboom D, Valkema R, Krenning EP. Radiolabelled peptides for tumour therapy: current status and future directions-plenary lecture at the EANM 2002. Eur J Nucl Med Mol Imaging. 2003;30:463-469.

6. Reubi JC, Waser B. Unexpected high incidence of cholecystokinin-B/gastrin receptors in human medullary thyroid carcinomas. Int J Cancer. 1996;67:644-647.

7. Reubi JC, Schaer JC, Waser B. Cholecystokinin(CCK)-A and CCK-B/gastrin receptors in human tumors. Cancer Res. 1997;57:1377-1386.

8. Behe M, Behr TM. Cholecystokinin-B (CCK-B)/gastrin receptor targeting peptides for staging and therapy of medullary thyroid cancer and other CCK-B receptor expressing malignancies. Biopolymers. 2002;66:399-418.

9. Smith CJ, Volkert WA, Hoffman TJ. Radiolabeled peptide conjugates for targeting of the bombesin receptor superfamily subtypes. Nucl Med Biol. 2005;32:733-740.

10. Brans B, Linden O, Giammarile F, Tennvall J, Punt C. Clinical applications of newer radionuclide therapies. Eur J Cancer. 2006;42:994-1003.

11. Brissette R, Prendergast JK, Goldstein NI. Identification of cancer targets and therapeutics using phage display. Curr Opin Drug Discov Devel. 2006;9:363-369.

12. Ruoslahti E. Drug targeting to specific vascular sites. Drug Discov Today. 2002;7:1138-1143.

13. Oyama T, Sykes KF, Samli KN, Minna JD, Johnston SA, Brown KC. Isolation of lung tumor specific peptides from a random peptide library: generation of diagnostic and cell-targeting reagents. Cancer Lett. 2003;202:219-230.

14. Ninck S, Reisser C, Dyckhoff G, Helmke B, Bauer H, Herold-Mende C. Expression profiles of angiogenic growth factors in squamous cell carcinomas of the head and neck. Int J Cancer. 2003;106:34-44.

15. Schmidt K, Hoffend J, Altmann A, et al. Transfer of the sFLT-1 gene in Morris hepatoma results in decreased growth and perfusion and induction of genes associated with stress response. Clin Cancer Res. 2005;11:2132-2140.

16. Eisenhut M, Mier W. Radioiodination chemistry and radioiodinated compounds. In: Vértes A, Nagy S, Klencsár Z, eds. Handbook of Nuclear Chemistry. Vol 4. Boston, MA: Kluver Academic Publishers; 2003:257-278.

17. Kuhnast B, Bodenstein C, Haubner R, et al. Targeting of gelatinase activity with a radiolabeled cyclic HWGF peptide. Nucl Med Biol. 2004;31:337-344.

18. George AJ, Lee L, Pitzalis C. Isolating ligands specific for human vasculature using in vivo phage selection. Trends Biotechnol. 2003;21:199-203.

19. Nanus DM. Of peptides and peptidases: the role of cell surface peptidases in cancer. Clin Cancer Res. 2003;9:6307-6309.

20. Wester HJ, Kessler H. Molecular targeting with peptides or peptide-polymer conjugates: just a question of size? J Nucl Med. 2005;46:1940-1945. 